\title{
Network Performance Metrics for Energy Efficient Scheduling in Wireless Sensor Networks (WSNs)
}

\author{
Felicia Engmann $\mathbb{D}^{1,2}$ Kofi Sarpong Adu-Manu $\mathbb{D}^{\mathbb{D}},{ }^{2}$ Jamal-Deen Abdulai, $^{2}$ \\ and Ferdinand Apietu Katsriku ${ }^{2}$ \\ ${ }^{1}$ School of Technology, Ghana Institute of Management and Public Administration, Accra, Ghana \\ ${ }^{2}$ Department of Computer Science, University of Ghana, Legon, Accra, Ghana \\ Correspondence should be addressed to Kofi Sarpong Adu-Manu; ksadu-manu@ug.edu.gh
}

Received 21 May 2021; Revised 26 July 2021; Accepted 9 November 2021; Published 27 November 2021

Academic Editor: Ihsan Ali

Copyright (c) 2021 Felicia Engmann et al. This is an open access article distributed under the Creative Commons Attribution License, which permits unrestricted use, distribution, and reproduction in any medium, provided the original work is properly cited.

\begin{abstract}
In Wireless Sensor Networks, sensor nodes are deployed to ensure continuous monitoring of the environment which requires high energy utilization during the data transmission. To address the challenge of high energy consumption through frequent independent data transmission, the IEEE $802.11 \mathrm{~b}$ provides a backoff window that reduces collisions and energy losses. In the case of Internet of Things (IoTs), billions of devices communicate with each other simultaneously. Therefore, adapting the contention/ backoff window size to data traffic to reduce congestion has been one such approach in WSN. In recent years, the IEEE $802.11 \mathrm{~b}$ MAC protocol is used in most ubiquitous technology adopted for devices communicating in the IoT environment. In this paper, we perform a thorough evaluation of the IEEE $802.11 \mathrm{~b}$ standard taking into consideration the channel characteristics for IoT devices. Our evaluation is aimed at determining the optimum parameters suitable for network optimization in IoT systems utilizing the IEEE $802.11 \mathrm{~b}$ protocol. Performance analysis is made on the sensitivity of the IEEE $802.11 \mathrm{~b}$ protocol with respect to the packet size, packet delivery ratio (PDR), end-to-end delay, and energy consumption. Our studies have shown that for optimal performance, IoT devices using IEEE $802.11 \mathrm{~b}$ channel require data packet of size 64 bytes, a data rate of $11 \mathrm{Mbps}$, and an interpacket generation interval of 4 seconds. The sensitivity analysis of the optimal parameters was simulated using NS3. We observed PDR values ranging between $27 \%$ and $31 \%$, an average end-to-end delay ranging within $10-15 \mathrm{~ms}$ while the energy remaining was between 5.59 and 5.63Joules. The results clearly indicate that scheduling the rate of packet generation and transmission will improve the network performance for IoT devices while maintaining data reliability.
\end{abstract}

\section{Introduction}

The proliferation of the Internet has seen an increase in the growth of the Internet of Things (IoT). There is a continuous flow of data seamlessly from trillions of IoT devices that usually go unnoticed and unused. It is expected that by 2021, 28 billion devices will be connected through IoT. IoT devices autonomously form a network that communicates with other devices continuously, hence generating massive amount of data from their deployable environment. IoT devices are adopted for earthquake, healthcare, vehicular tracking, and agricultural monitoring applications. IoTs are similar to Wireless Sensor Networks (WSNs) in their operations. They are made up of a large number of small batteryoperated devices that can sense, process, and communicate data wirelessly [1]. Their key benefits are their ability to operate in harsh environments unattended, where human control schemes are difficult or infeasible to implement [2]. The use of IoTs and WSNs has been proven superior to the traditional methods of collecting data from the environment. With IoTs and WSNs, sensor nodes autonomously form interconnected networks that collaborate in data collection [3]. However, 
sensor devices can replace traditional devices by sending sensed data to sinks through single-hop or multihop communication. IoT devices mostly communicate via IEEE 802.11 standard due to its flexibility of implementation and scalability.

IoT devices transmit data from different sources such as wearable devices, smart vehicular systems, smartphones, and several laptops. All these devices generate data transmitted through cellular base stations, Wi-Fi access points, and other Road Side Units (RSUs) to a service provider. There are a huge number of transmissions received at each access point creating packet losses due to the limited channel access. Hence, the need to design communication protocols resolves the channel access problems.

The communication protocols designed for IoT and WSN applications sought to address challenges related to collision, overhearing, protocol overheads, and idle listening. The collision occurs when multiple nodes attempt transmitting packets at the same time. The simultaneous multiple transmission from IoTs and WSNs increases the reception cost at the destination node while increasing the cost of retransmission in the source node. Nodes usually listen for transmission on the channel to avoid collisions. Nevertheless, when nodes stay awake listening multiple times for packets destined for another node, overhearing occurs. In overhearing, the broadcast of packets by the wireless medium causes all one-hop neighbors (within its range) of the source node to hear the transmitted packet, aggravating overhearing [4].

Nodes in an idle mode listen to the channel awaiting possible incoming packets. However, a sensor node in an idle state can consume a similar amount of energy as a node that is receiving a packet [5-7]. As a result, turning off the transceiver in idle mode is critical to reducing excessive energy consumption in IoTs and WSNs $[2,8]$. However, frequently turning off the transceiver to sleep introduces longer latencies and increase in the exchange of overhead packets. A number of authors have proposed Time Division Multiple Access (TDMA) approaches to mitigate the challenges of overhearing, collision and idle listening [2]. However, an essential factor in TDMA-based protocol is when to update the transmission schedule to prevent conflicts in transmissions from neighboring nodes. Since enforcing fixed transmission schedules might not have proven energy-efficient for networks requiring continuous monitoring, scheduling based on the spatiotemporal differences of data from deployed sensor nodes is proposed and the simulation results are presented in [9].

However, medium access control (MAC) protocols implement some scheduling that allows nodes to communicate in a way that prevents multiple collisions and energy wastage. The IEEE 802.11b MAC protocol defines a standard physical and MAC layer that specifies the access protocol for all nodes in the network. The Distributed Coordination Function (DCF), one of two coordination functions in the MAC layer, supports asynchronous transmissions which as supported in IoT environments [10].

This paper, therefore, evaluates the traditional MAC protocol implemented in the IEEE $802.11 \mathrm{~b}$ that schedules nodes to sleep and wake up within some optimum periods.
Moreover, the MAC protocol of the IEEE 802.11b implements the DCF which mandates a gap of specified minimum delay. The DCF implements a binary exponential back-off the carrier sense multiple access with collision avoidance (CSMA/CA) for effective implementation in both ad hoc and infrastructure systems. Due to the number of simultaneous transmissions, scheduling transmissions of individual nodes is a challenge in IoTs. In this paper, we examine the effect of network parameters such as the payload size, the packet interarrival interval, and the data rate of the IEEE802.11b.

The remaining sections of the paper are discussed as follows. Section 2 presents energy management issues in WSNs. In Section 3, we present related works. In Section 4, simulation results and discussions on the sensitivity analysis of IEEE802.11b are provided. Finally, Section 5 concludes the paper.

\section{Energy Management in WSN}

In WSNs, sensor nodes sample data from the environment and wirelessly transmit to a base station for onward processing and the outcome communicated via the Internet to remote users. To ensure the continual availability of communication between the sensor nodes and the base station, energy management of the various components of the sensor node, illustrated in Figure 1, is paramount as presented in [11]. However, despite the implementation of these energy management principles, the energy is consumed by the communication subsystem of the sensor node, mainly controlled by the radio, the primary source of energy consumption during transmission as shown in Figure 2.

Energy management includes energy harvesting, energy transfer, and algorithmic schemes (protocols). These energy management approaches restore depleted energy in a sensor node. The rate of discharge of energy through the operations of the radio is mainly faster than the rate of energy replacement by the energy management approaches mentioned in the text. The rate of energy depletion in sensor node makes continuous communication a significant threat to the continuous operation in WSN applications [11]. There is, therefore, the need to control the frequency of communication to reduce the amount of energy consumed by the sensor node, especially, during data transmission.

Duty cycling, an energy conservation method (as represented in Figure 3), schedules the sensor nodes to be turned on or off at regular intervals to reduce the frequency of the operations of the sensor nodes. However, the operations of the sensor node require a trade-off between energy efficiency on one side and latency and throughput gains on the other. To capitalize on the benefits of duty cycles, the authors in [5, 12] proposed power management, topology controls and MAC layer algorithms, and dynamic sleep wake up cycles $[13,14]$. However, challenges of regular sleep/wake-ups include the exchange of extra overheads, data losses, and increased latencies and associated energy losses. The possibility of mitigating these challenges includes reducing the frequency of switching to sleep if the node's sleep and wake-up schedule are not mainly based on the network activity [5]. 


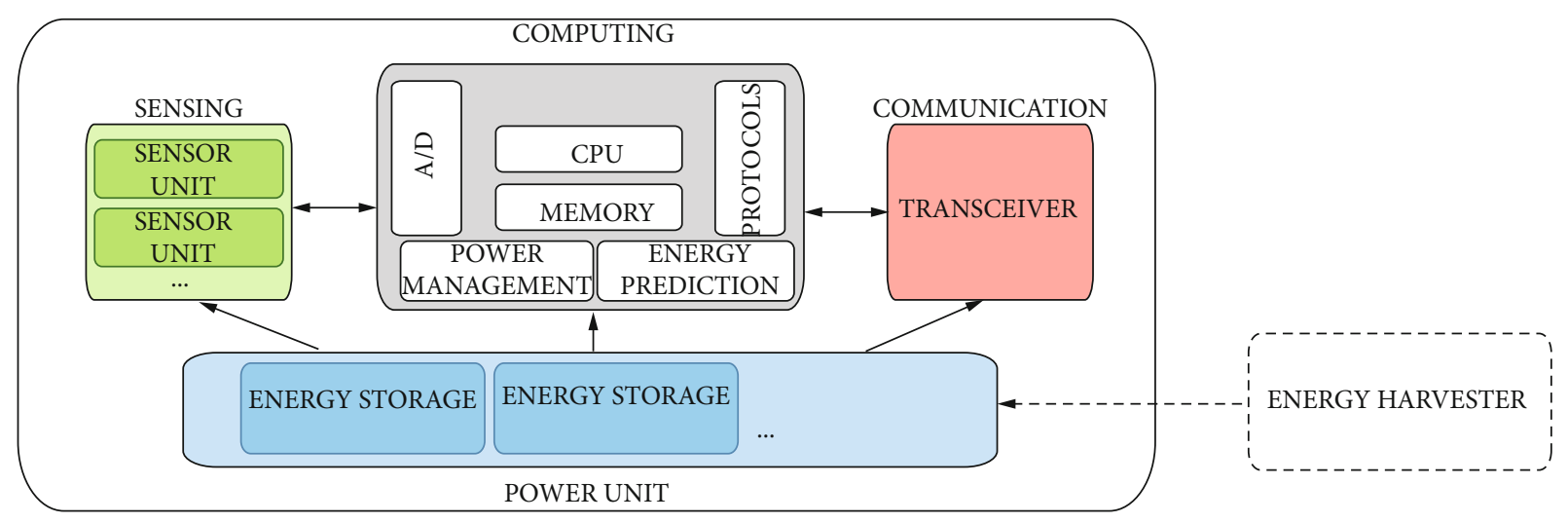

FIgURE 1: Wireless sensor node.

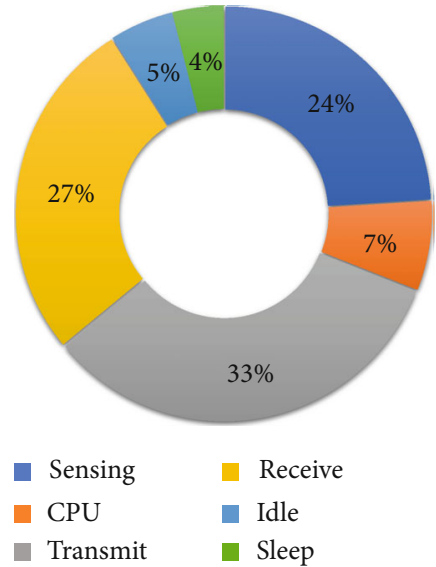

FIGURE 2: Energy consumption in WSNs.

IoTs introduce a unique challenge of several packets being transmitted simultaneously from randomly placed nodes which may introduce multiple interferences if care is not taken. However, these IoTs have the opportunity to learn and adapt their radios to the activities of its neighboring nodes. An interference and spectrum aware channel access mechanism was proposed by authors in [10]. The channel access approach was proposed because DCF, Point Coordination Function (PCF), and Time Division Multiple Access (TDMA) could not prove efficient in managing interference.

Meanwhile, the DCF implementation with CSMA/CA used in the IEEE $802.11 \mathrm{~b}$ has been evaluated to decide on optimum parameters for network implementation in the face of these known challenges [15]. In their work, simulations of the DCF with basic mechanisms assumed that nodes arrive in a Poisson distribution interval with fixed payload sizes. However, due to the parameters of the IEEE $802.11 \mathrm{~b}$ channel, these channel conditions require some standard network parameters to obtain optimum results. These optimum network output include a decrease in the channel delay, increased throughput, and reduction in the energy consumed.

MAC protocols with low duty cycle implementation in WSNs may be classified as either contention-based or TDMA. The energy saving occurs when deployed nodes largely remain in inactive modes until the detection of events. However, when several nodes in proximity detect events and wake-up to sense and transmit, without proper scheduling, extensive collision may occur. Strategies implemented in literature to overcome the challenges of scheduling in WSNs have been implemented in the literature. One such approach is the Sensor MAC (S-MAC), a scheduling method that allows border nodes to adopt a diversified transmission schedule in WSNs [16]. The approach used in SMAC is to mitigate the high energy lost due to the switching of border nodes in virtual clusters. The rational of the one-time scheduling implementation is to reduce the energy the sensor node spends in its frequent switch between listening and sleep mode. TDMA approaches have also been implemented. One of such implementations proposes a tight-time scheduling and increased throughput scheduling with TDMA [17]. The scheduling method reduced the energy and overhead costs incurred by the network during the network setup phase. However, much time and overhead costs were spent in the initial network setup phase. Another TDMA technique was implemented for fast data aggregation at fixed intervals for scheduling when frequent transmissions are required [2]. Since most TDMA implementations require a fixed interval of transmission, the data itself has less effect on the schedule of its packet transmissions. Most TDMA systems are also not effective for larger network sizes since scalability of the scheduling is a challenge. The regular intervals of TDMA, also, introduce several unused slots that increase latency. In our approach, we do not employ the use of tight time synchronization (TTS) for node transmission. Nodes have equal chance to compete for channel access, but due to the limited number of competing nodes accessing the channel, our approach overcame the challenges of collision that TTS is designed to solve.

Since the CSMA/CA protocol implementation of DCF in IEEE $802.11 \mathrm{~b}$ also introduces some basic scheduling, the paper is aimed at obtaining network parameters that could optimize transmissions of multiple packets when no further scheduling is used up.

\section{Related Works}

In this section, we provide related works that focus on an access method in IEEE802.11b. Many wireless devices in 


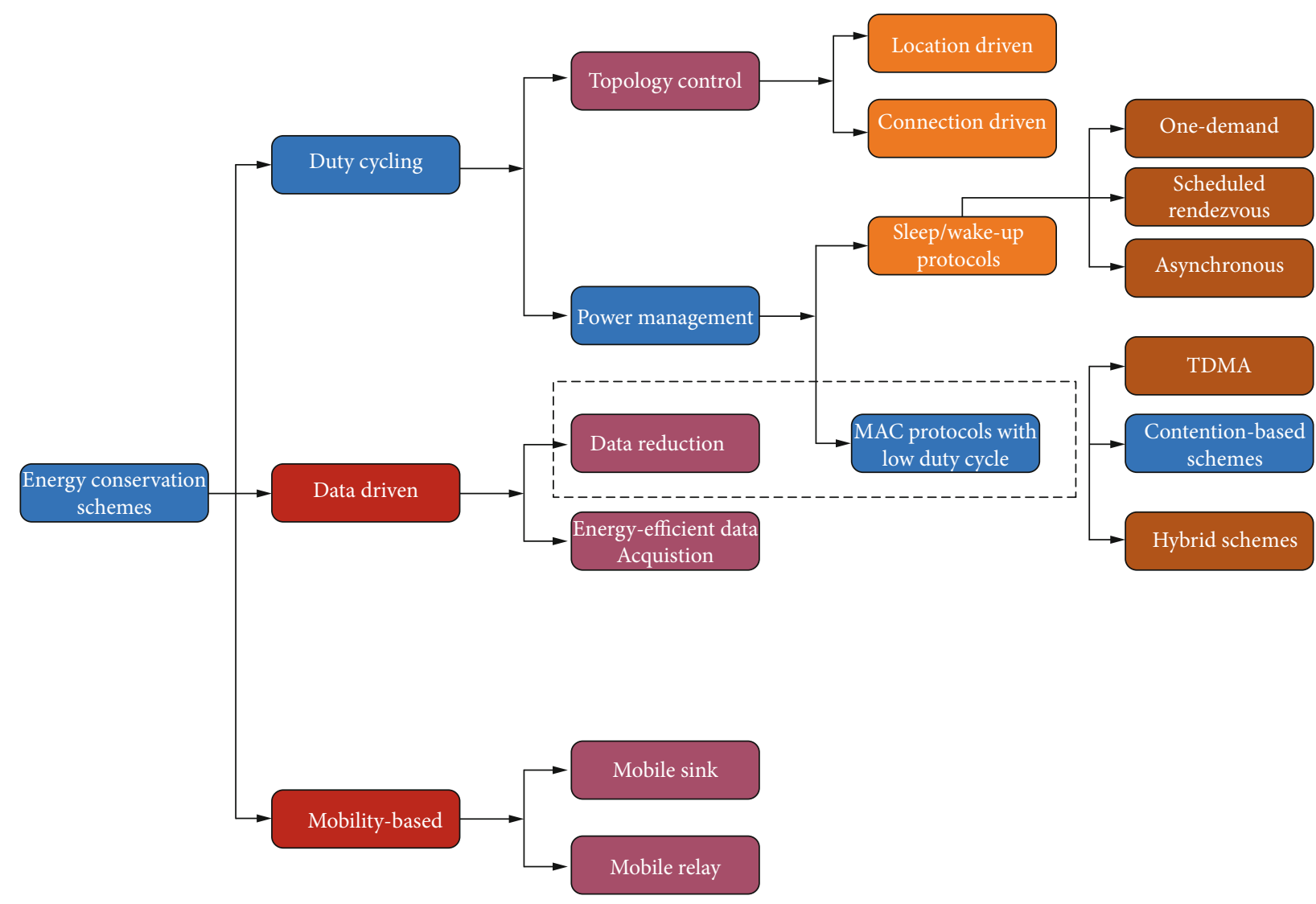

FIGURE 3: Energy conservation schemes.

the case of IoTs create a major bottleneck in the wireless channel during the data communication process. Early studies on heavily utilized IEEE802.11b channel were performed to optimize the network performance using link layer information [18]. Congestion in the channel directly impacts on its use; therefore, the authors observed that RTS/CTS which prevents congestions may conflict with node's fair access to the shared channel. Also, even though IEEE802.11b provide users with 4 data rates, which include $1 \mathrm{Mbps}$, $2 \mathrm{Mbps}$, $5.5 \mathrm{Mbps}$, and $11 \mathrm{Mbps}$, users frequently use the $1 \mathrm{Mbps}$ and $11 \mathrm{Mbps}$ channels. In their work, the authors highlighted the detrimental effect of rate adaptation of channels to network performance if implemented as a response to congestion.

Some researchers have proposed the optimization of the IEEE802.11b channel through adjusting the contention window size $[16,17]$ or the frame sizes [19-21] in relation to the traffic in the channel. The DCF implementation in $802.11 \mathrm{~b}$ also suggests the implementation of multirate adaptation channels to mitigate the poor throughput performance of the low data rate channels.

The basic contention-based protocol of MAC is the DCF which is implemented in most WSNs due to its simplicity of implementation, ability to counter the hidden terminal problem, and scalability as seen in IoTs.

In the operation of the DCF Protocol in CSMA/CA, nodes willing to transmit wait a predefined DCF Interframe Space (DIFS) of $50 \mu \mathrm{s}$. If the transmitting node's physical layer does not sense any signal within the DIFS, a clear channel assessment (CCA) is sent to the MAC layer. If the recipient node does not sense any packets in transition, it waits a random period known as the contention window. If no signal is sensed, the transmitting node sends a Request-to-Send (RTS) to the receiving node. The RTS contains the MAC Address of the transmitter and receiver. It also has a field that contains the duration of the first MAC fragment. The RTS information allows other transmitters to determine their Network Allocation Vector (NAV) to prevent collisions. Hence, in our work, we consider an optimum interpacket interval that might influence the number of packets that will contend for the channel at every point in time. The receiver node sends a Clear-to-Send (CTS) signal after a short inter-frame space (SIFS) of $10 \mu \mathrm{s}$, which contains information that adjusts the neighbor NAV. When a packet is too large, it is divided into several MAC fragments which will be successively sent to the recipient. A diagram of the transmissions of two nodes depicting the explained process above is presented in Figure 4. The work done in this paper, therefore, seeks to also find an optimum payload size combined with its interpacket interval and prevents congestion of the channel if several packets need to be sent in an IoT environment.

A flowchart of the CSMA/CA is presented in Figure 5. The basic CSMA/CA with DCF that coordinates node operation in ad hoc mode permits all nodes to communicate within their transmission range without enforcing association and beacon generation. Because no association rules 


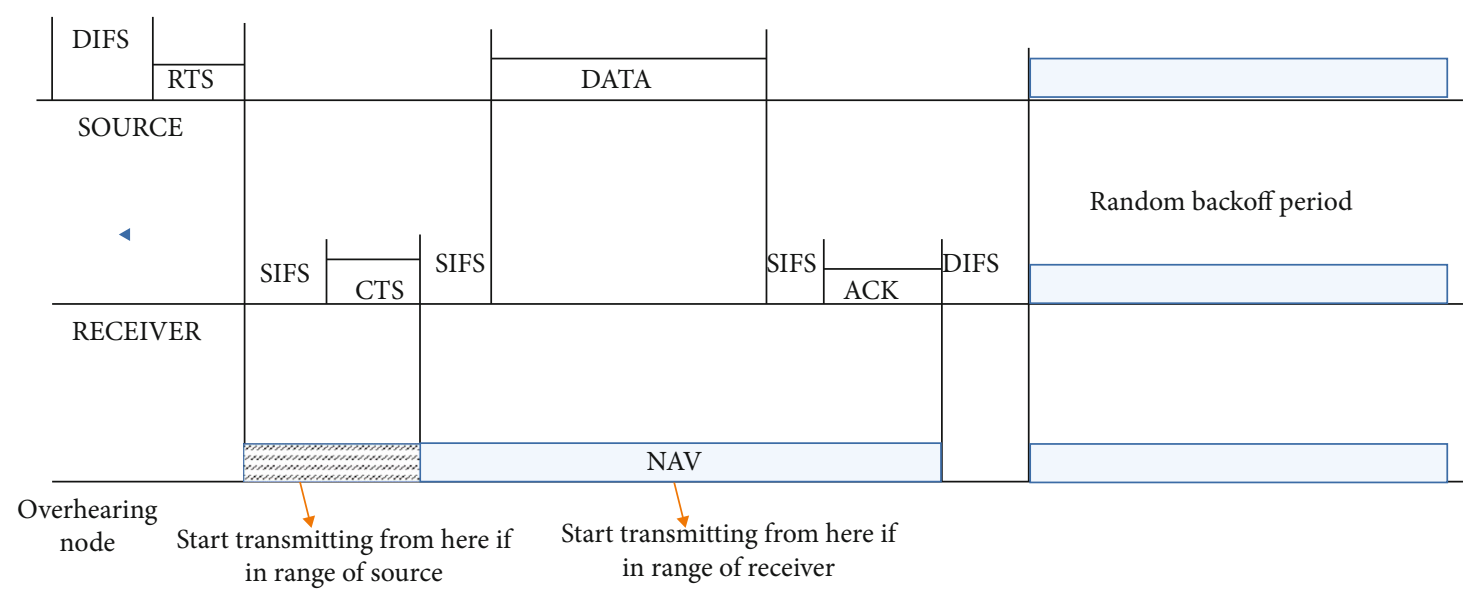

FIgUre 4: Two devices communicating on an IEEE $802.11 \mathrm{~b}$ channel with CSMA/CA.

are enforced to coordinate communication, nodes always remain ready to receive messages from neighbors. Hence, nodes in this infrastructure do not sleep but are in a constant idle power consumption state.

\section{Simulations and Discussions}

An IoT environment typically may have several devices transmitting data to a single receiving device. The energy parameters that relate to the different states of a typical Micaz node used in this paper's simulations are presented in Table 1.

An illustration of a typical IoT system where a number of nodes communicate via a common channel to a sink node is also presented in Figure 6. We observe that the unregulated communication emanating from the sensor nodes causes collision in the channel. Hence, the data arriving at the sink node may be greatly distorted.

From Figure 6, only one node [i.e., node 1] will have access to the channel at a time. The remaining generated packets arriving from nodes 2 to 5 may collide and drop since the channel is busy and not available. The channel is saturated when the queue has more than some maximum value or maxSize of packets. Therefore, any additional packets will be dropped. Packets may also be dropped when they stay longer than Dmax in the channel when competing for channel access, which in IEEE 802.11b implements as FIFO queue.

For our simulations, we assume a first-in-first-out (FIFO) queue implementing a maxSize of 500 packets and a Dmax of 500 seconds (maxSize is the maximum number of packets that can be the queue while Dmax is the maximum time a packet may remain in the queue). The queuing process of the channel as shown in Figure 7 depicts the interval between successive packets en-queuing. The number of packets in the queue may be reduced if the interval between successive en-queuing increases, such that the rate of de-queuing is faster than the interval for en-queuing. Hence, with appropriate scheduling, the optimum number of nodes will occupy the channel within a Dmax while preventing excess collisions.
To obtain the optimum combination of the payload/ packet size and interval, the simulations also considered the energy consumption of the nodes. Network performance metrics used included the average end-to-end delay, packet delivery ratio, and energy consumption. The simulations performed on the IEEE $802.11 \mathrm{~b}$ channel are to generate these optimum parameters to enable a scheduling implementation that reduces the number of packets in the channel per unit time while avoiding collisions and reducing latencies.

4.1. Simulation Parameters. Table 2 shows the network parameters used in the simulation. The NS3 simulator, a discrete event simulator with MAC layer and extensive energy support, implements the scheduling algorithm proposed. Each sensor node communicates with each other directly within its communication range.

General assumptions made on the network include a fixed number of sensor nodes randomly deployed in a fixed-sized network. All nodes are homogenous in size, capabilities, and initial energy. For a single-hop network, all nodes are within a $100 \mathrm{~m}$ distance away from the sink. Nodes are not GPRS enabled; hence, they assume the distances from each other using received signal strength indication- (RSSI-) based methods. The simulations adopt the IEEE802.11b channel, corresponding to the likely channel for IoTs. We assume an omnidirectional antenna and suppose there will be no fast or slow fading antenna signal. The network has randomly deployed nodes transmitting directly to a single sink, as shown in Figure 8. Nodes are not required to synchronize with each other or the sink before initiation of communication. Parameters for simulations are as presented in Table 2.

4.2. Simulation Results of the Network Performance of the IEEE802.11b. The primary network parameters used were 32-bytes of payload size transmitted at a data rate of $11 \mathrm{Mbps}$; simulation time is $1000 \mathrm{~s}$ at a packet generation rate of $1 \mathrm{~s}$. The network assumes implementation of the IPV4 base MAC of 255.255.255.0, and hence a maximum of 254 nodes are enough to perform investigations for the simulations. For multiple nodes connected to a single 


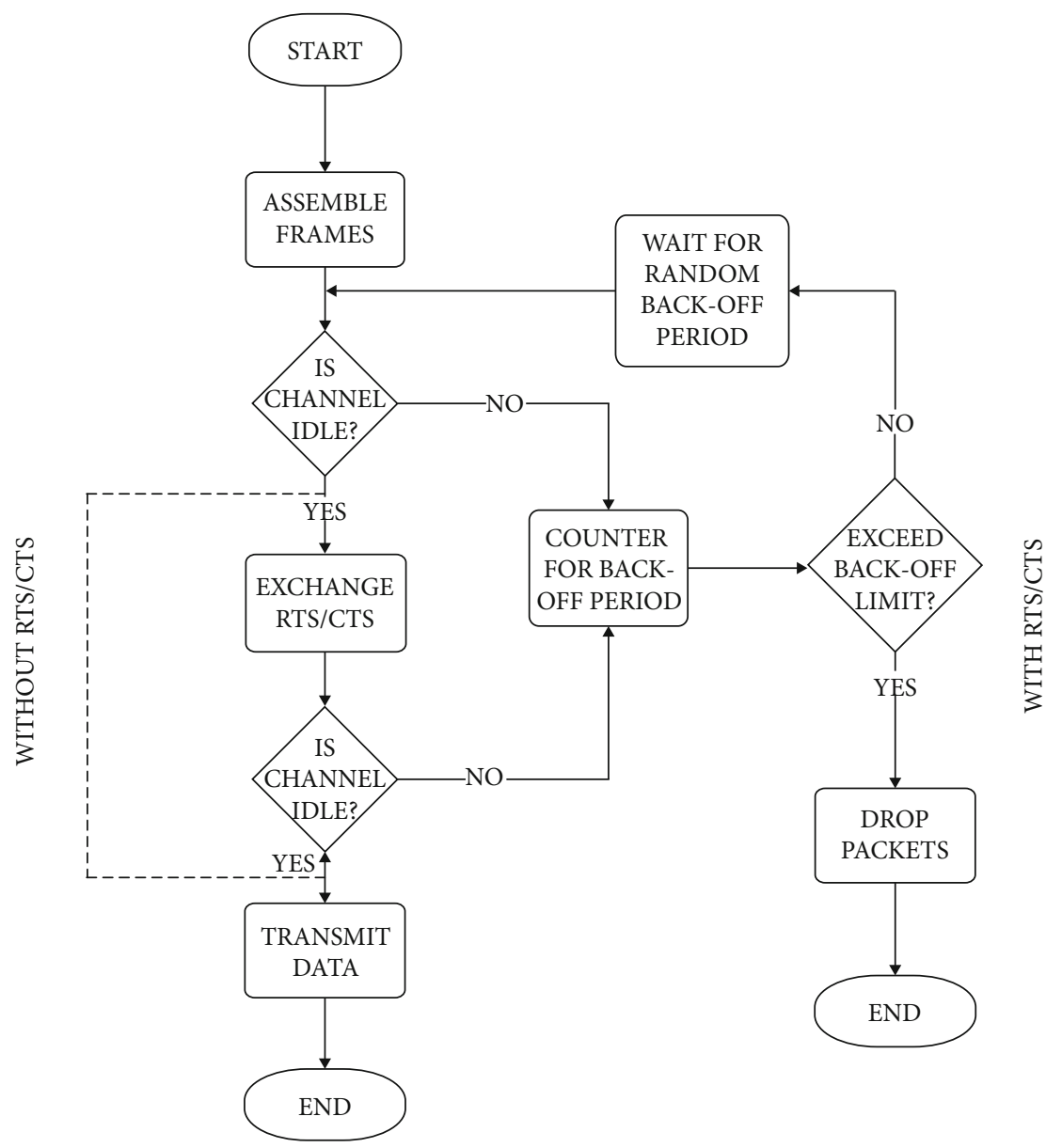

FIGURE 5: CSMA/CA in IEEE802.11b.

TABLE 1: Energy measurement of IEEE802.11 used in the simulations $[3,22]$.

\begin{tabular}{lcc}
\hline State & Current draw (A) & Power consumption in Watts \\
\hline TX & 0.380 & 1.14 \\
RX & 0.313 & 0.94 \\
IDLE & 0.273 & 0.82 \\
SLEEP & 0.033 & 0.10 \\
\hline
\end{tabular}

destination, queuing on the channel may be one of the principal causes of collisions that affect network performance.

4.3. Effect of Data Rate on the Node Density. The nodes are deployed randomly in a river sensor network (RSN) of area 100 by 100 square meters. We assume that the deployed nodes are on the surface of the river and make little or no movement, therefore modelled as static. Nodes deployed are assumed to be in the one-hop communication range of the sink. If the communication range is assumed to be $100 \mathrm{~m}$, any node placed within this area will be successfully transmitted without using intermediary nodes for multi hopping. Sensed data is transmitted immediately without an intentional delay; hence, the MAC is assumed to be in an ad hoc mode. Comparing the different data rates of
IEEE802.11b, simulations run for a period of 1000 seconds with a seed run of 1000 . The initial energy on the nodes is $20 \mathrm{~J}$, and the interpacket generation interval is 1 second.

Observations of the graph in Figure 9 indicate simulations for data rates $11 \mathrm{Mbps}, 2 \mathrm{Mbps}, 5.5 \mathrm{Mbps}$, and $1 \mathrm{Mbps}$, recording an increase in the average end-to-end delay of packets generated over the increasing node densities. $1 \mathrm{Mbps}$ channel records the highest delay for all node densities. The delay ranges between $14 \mathrm{~ms}$ at the highest and about $10 \mathrm{~ms}$ between 140 and 160 nodes. The $11 \mathrm{Mbps}$ channel generally has the best delay. Its average end-toend delay values range between $11 \mathrm{~ms}$ at the highest and $9.8 \mathrm{~ms}$ at its lowest. The recorded delay indicates more collisions in the narrow channels of $1 \mathrm{Mbps}$ as opposed to the $11 \mathrm{Mbps}$ channel. The time it takes a packet to access the channel and the retransmission delay are the main factors causing delays in WSN. All data rates have their average delays recording closer values when the network has between 140 and 160 nodes.

The increase in packets causes a corresponding increase in the traffic per unit time. Since the slot time for $802.11 \mathrm{~b}$ is $20 \mu \mathrm{s}$, an increase in the number of nodes increases the competition for the channel. The resulting increase causes collisions and retransmissions, increasing the possible number of dropped packets. The average PDR for 1, 2, 5.5, and 


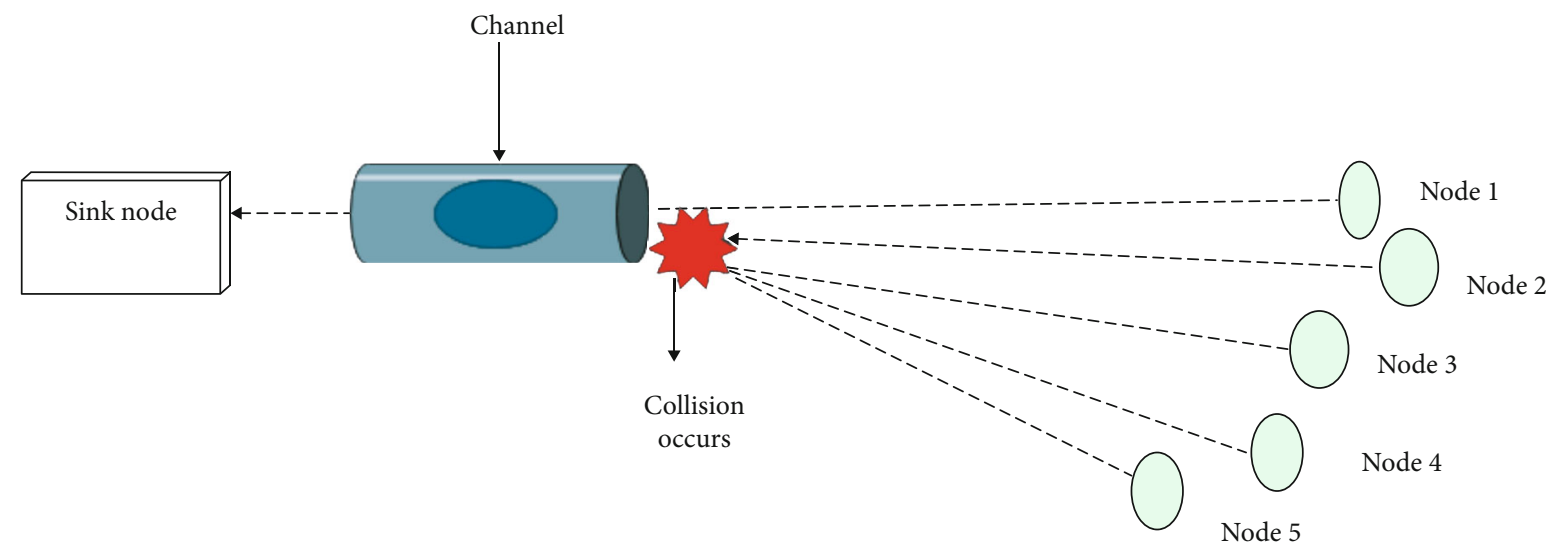

FIGURE 6: Link level collision for multiple nodes directly communicating to a single source.

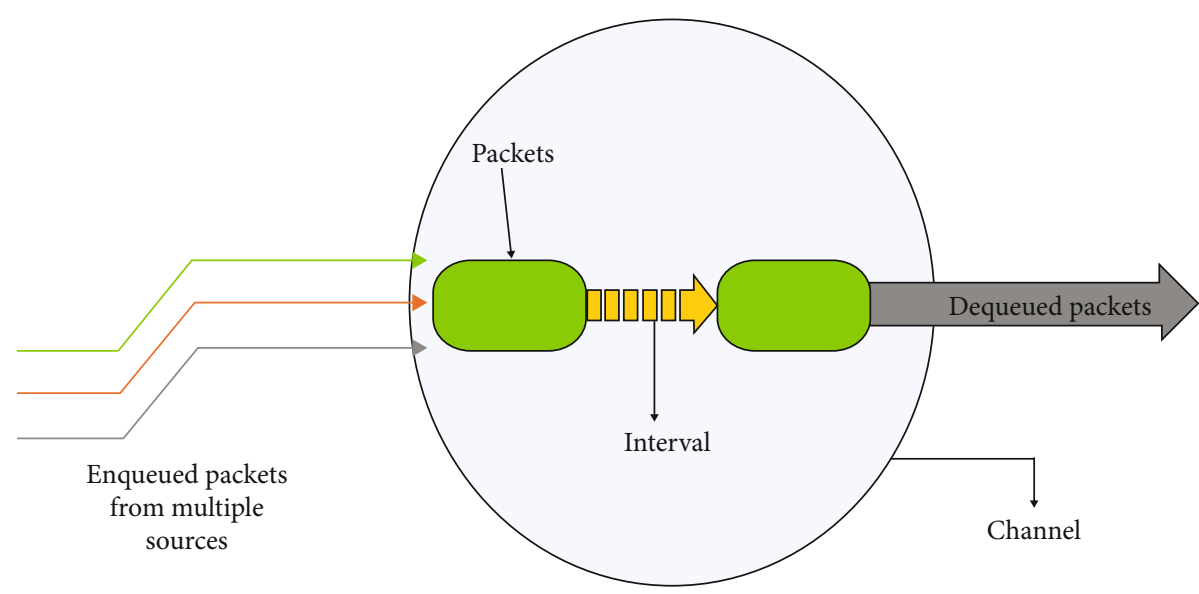

FIGURE 7: Link level queuing for multiple sources communicating directly with sink.

TABLE 2: Network simulation parameters.

\begin{tabular}{|c|c|c|}
\hline & Network parameters & Value \\
\hline \multirow{3}{*}{ Network parameters } & Number of nodes & Between 2 and 250 \\
\hline & Number of sink & 1 \\
\hline & Initial energy on node & $20 \mathrm{~J}$ \\
\hline \multirow{5}{*}{ Packet parameters } & Number of packets & Unlimited \\
\hline & Number of retransmissions & Max 7 \\
\hline & Packet size & $32,64,128,512,1024$ bytes \\
\hline & Packet interval & Between 0.5 and 5 seconds \\
\hline & Traffic type of packets & CBR \\
\hline \multirow{2}{*}{ Communication parameters } & Sensor communication range & $100 \mathrm{~m}$ \\
\hline & Data rate & $1 \mathrm{Mbps}, 2 \mathrm{Mbps}, 5.5 \mathrm{Mbps}$ and $11 \mathrm{Mbps}$ \\
\hline \multirow{4}{*}{ Transmission slot parameters } & Slot time & $20 \mu \mathrm{s}$ \\
\hline & Queue length & 50packets \\
\hline & Slot length & \\
\hline & Slot duration & 20 microseconds (for $802.11 \mathrm{~b}$ ) \\
\hline Number of seed runs & & 1000 \\
\hline Simulation time & & Max 1000secs \\
\hline
\end{tabular}




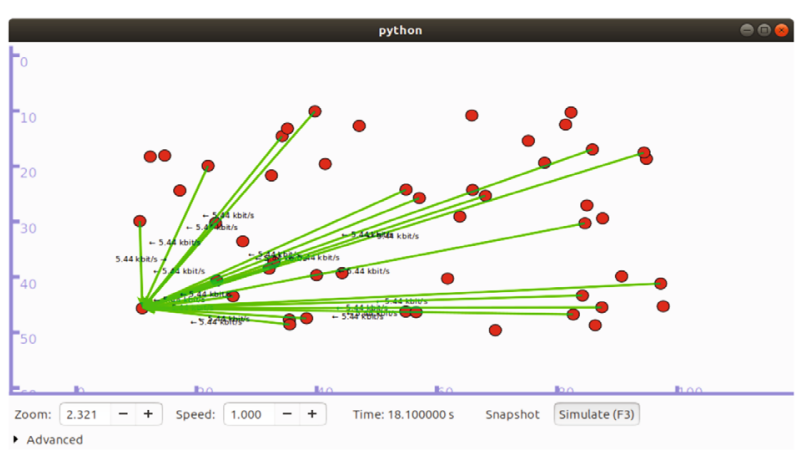

FIGURE 8: Randomly deployed stationary nodes communicating to a stationary sink.

11 Mbps channels are $8.667,13,15.2$, and 17.8 , as illustrated in Figure 10.

The causes of energy waste for ad hoc MAC include collisions that result in retransmissions of the collided packets and idle listening, which occurs when nodes listen in vain to receive packets. WSNs generally operate in the idle modes for more extended periods and transmit during its active state of the duty cycle. Nevertheless, the energy consumption of the idle state is almost the same as energy for transmission/reception of data. At the same time, it is much higher than the energy consumed during the sleep mode. When the power of the battery source reduces below the energy threshold, nodes remain in an idle state. In the idle state, the node switches to a low-power state and turn-off their transceiver. Therefore, in an idle state, no transmission or reception of data is possible, but the node remains alive to listen to the channel.

When the network density is 140 nodes, the remaining energy on the nodes does not permit the further transmission of packets. The energy remaining permits the sink node to receive; hence generated packets may not be transmitted, reducing $\mathrm{PDR}$ and causing energy waste in the network. Observations from Figure 11 show that the $1 \mathrm{Mbps}$ channel has the least remaining energy, while the $11 \mathrm{Mbps}$ channel has the best energy consumption. This minimum energy results from collisions that generate retransmissions when the data rate of the channel is higher. In the next section, the interpacket generation time is varied.

4.4. Effect of Interpacket Generation Interval. Inter packet generation interval (IPI) is the difference between successive packets generated from the same source node in a network. For 2, 10, 50, 100, and 150 nodes, we compare some random intervals from 1 second and below. The PDR obtained is for intervals which are multiples of the delaythreshold of IEEE802.11b represented as Inter-Packet generation Intervals (IPI). For the same simulation period and node density, the ratio of packets transmitted to packets received when the IPI increases do not change significantly. The PDR ranges from $100 \%$ to $8 \%$ for 2 to 150 nodes, respectively. The effect of IPI on the ratio of packets received and transmitted was not significant in these minor interval differences. However, observations show that an IPI of 1 second gives the best PDR.
The PDR generally decreases with increasing network density, as nodes increase from 100 to 200 nodes. The highest PDR is recorded at 100 nodes when the IPI is 3 seconds. Unlike the PDR at 1 second, which is about $25 \%$, IPI of 3 seconds records a better PDR of $31 \%$. However, observation shows that the PDR for particular network density does not follow a regular pattern. However, the PDR is best for IPI between 3 and 4.5 for all network densities observed, as shown in Figure 12.

Comparing the average end-to-end delay, intervals of 0.5 and 1 performed better with most minor delays, as illustrated in Figure 13. The delay was $0.3 \mathrm{~ms}$ for 2 nodes and increases $10 \mathrm{~ms}$ at 100 nodes when the IPI is either $0.5 \mathrm{sec}$ onds or 1 second. On the other hand, 0.9 seconds recorded the highest delay at 100 nodes. The graph suggests that the network reaches saturation after 100 seconds. Further observations of node densities concentrate on nodes from 100 nodes and beyond.

0.5 seconds is the maximum threshold for average E2E delay in the channel; after which packets remaining in the channel are dropped.

The average remaining energy, presented in Figure 14, is dependent on the total number of nodes available in the network. Therefore, when the network is saturated and the node battery reaches its low energy threshold, the per-node remaining energy remains relatively stable for an increasing number of nodes. This regular graph represents the fairness of energy and load distribution during the network lifetime. However, energy consumption depends on the number of transmissions/reception of packets and other channel access conditions. The network saturates after 140 nodes; hence, the total remaining energy remains almost the same for all network densities. IPI of 1.5 and 3.5 seconds recorded the highest remaining energy for 100 nodes, but the remaining energy levels were highest for IPI between 3.0 and $5.0 \mathrm{sec}-$ onds for all network densities. This buttressed the trends observed in the PDR and Average E2E delay graphs that the network performance improves with higher IPI. However, the particular IPI chosen must be according to the network density.

4.5. Effect of Packet Size (Payload) on Network Performance. Generally, the energy consumption of a wireless network depends mainly on the transmission of packets and the network's reliability. Smaller packets may increase the chances of reliable channel transmissions since fewer errors may occur in the channel. However, smaller packet sizes increase the overhead of network protocols and error-correcting codes, leading to less energy-efficient transmissions. In WSN, the collision rates experienced during transmission are closely related to the size of the packet. Analysis in [19] suggests an increase in the payload size decreases the MAC layer failure rate.

The effect of the payload size on the network performance investigated using network densities of 100 by 20 nodes to 200 is shown in Figure 15. The best PDR is recorded in all network densities for payload size 64 bytes, while 32 bytes had the worst PDR. The observed trend confirms a decrease in MAC failure as packet size increases. 


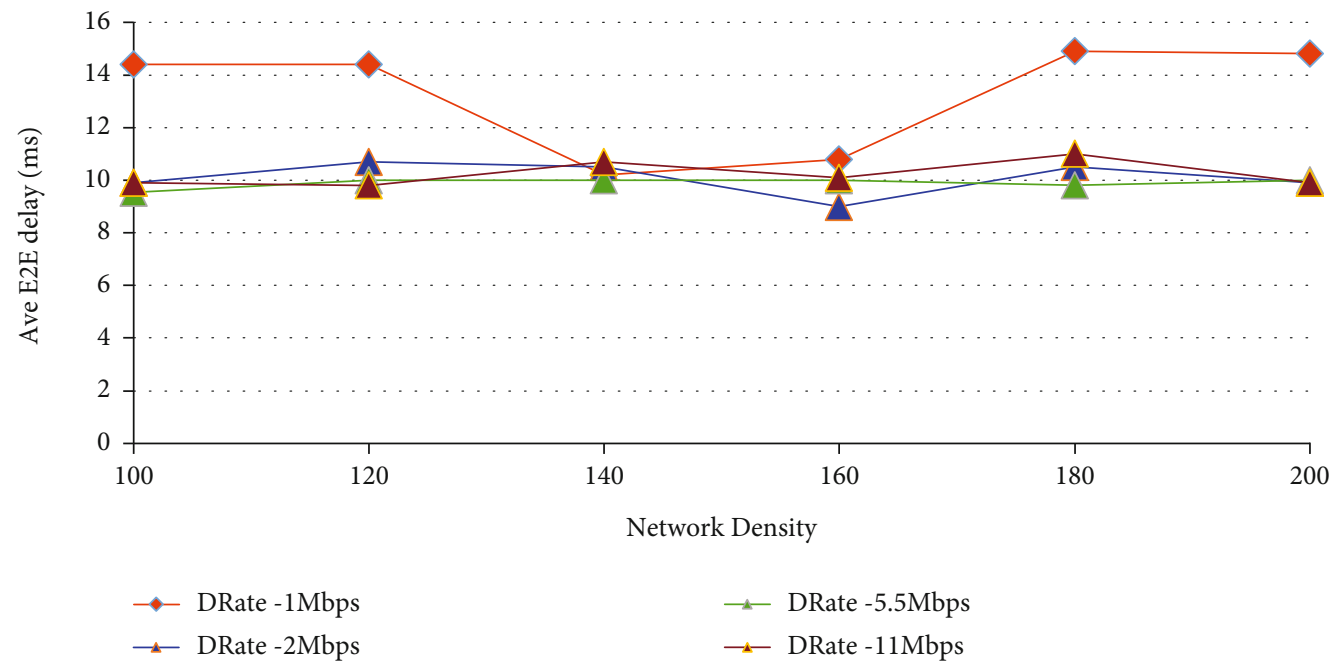

FIgUre 9: Average end-to-end delay of 1, 2, 5.5, and $11 \mathrm{Mbps}$ channel in IEEE 802.11b.

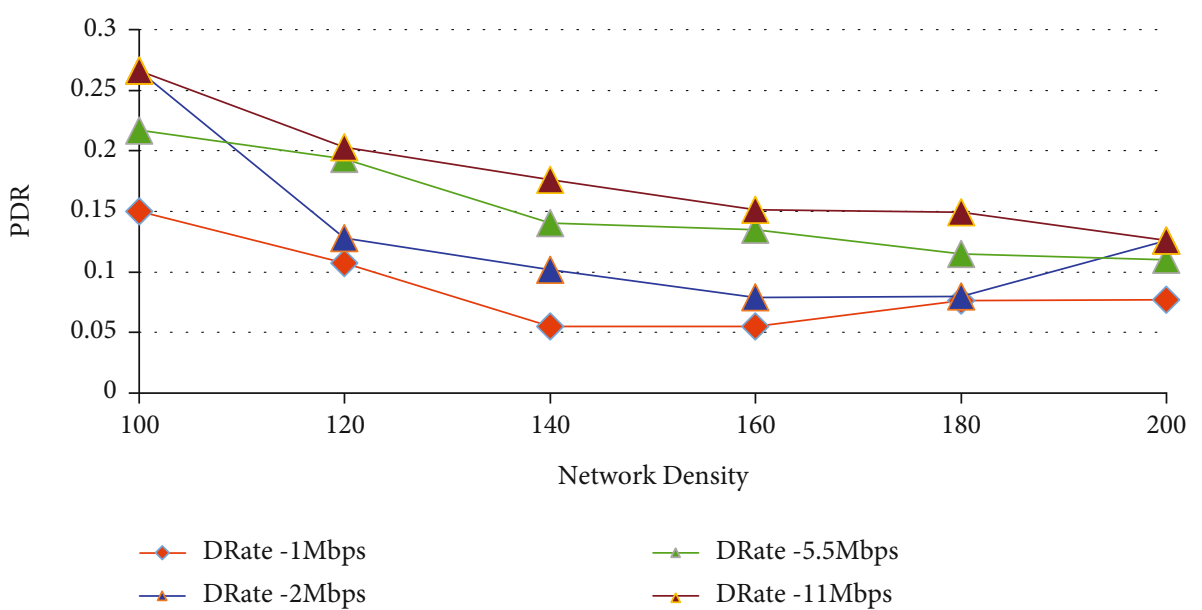

Figure 10: Packet Delivery Ratio of 1, 2, 5.5, and $11 \mathrm{Mbps}$ channel in IEEE 802.11b.

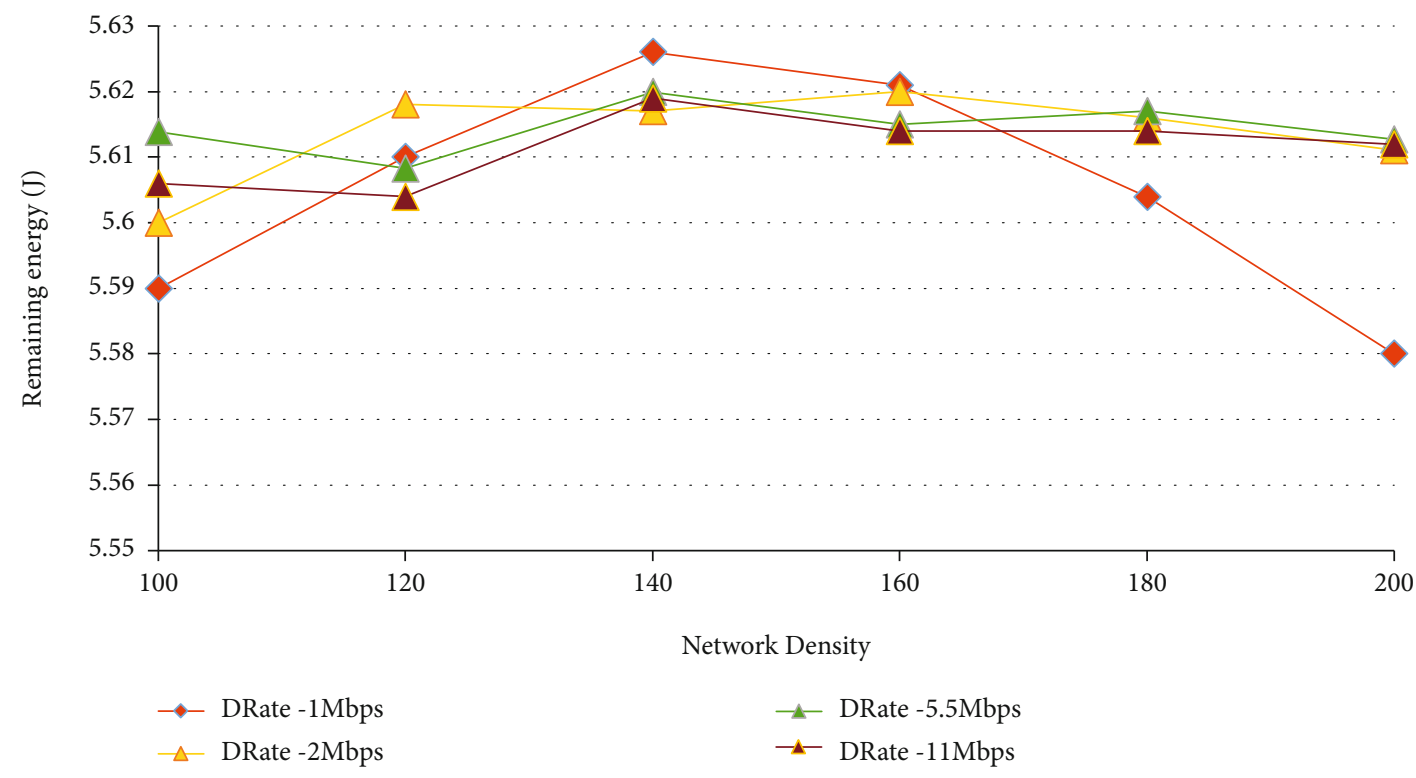

FIgURE 11: Remaining Energy of the 1, 2, 5.5, and $11 \mathrm{Mbps}$ channel in IEEE 802.11b. 


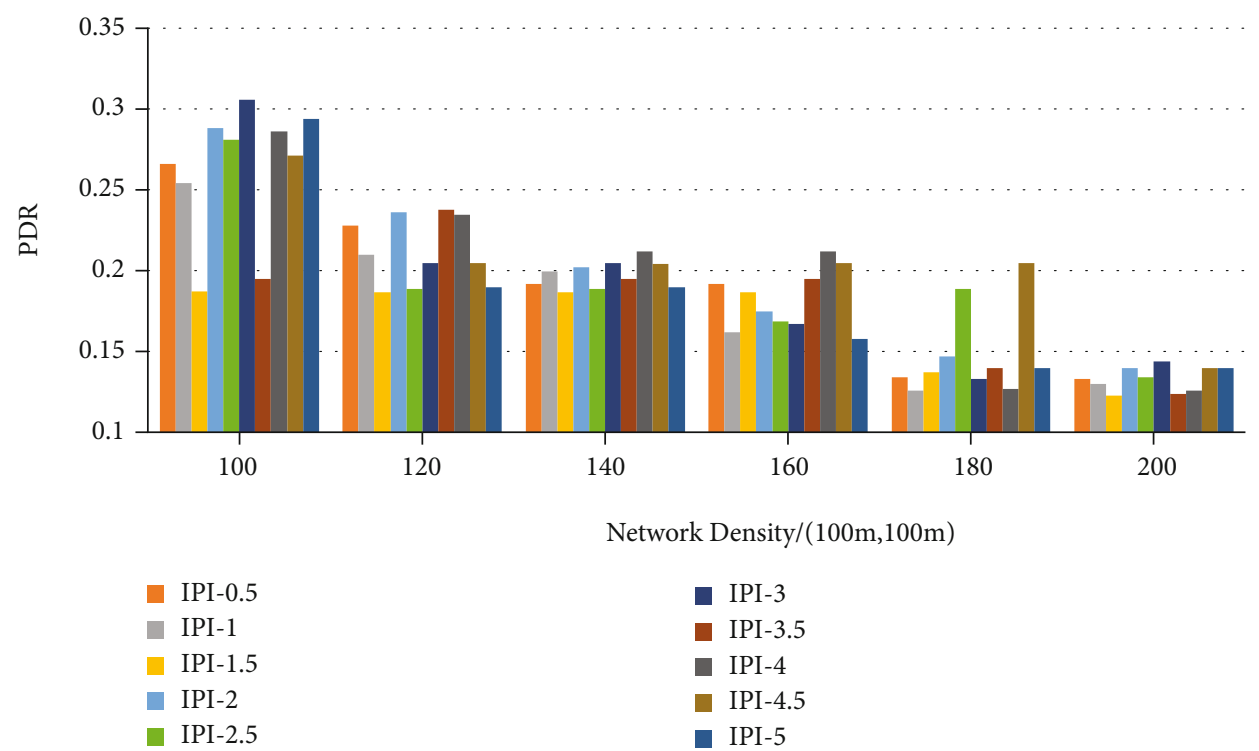

Figure 12: Packet delivery ratio of interpacket generation interval over 100 to 200 nodes.

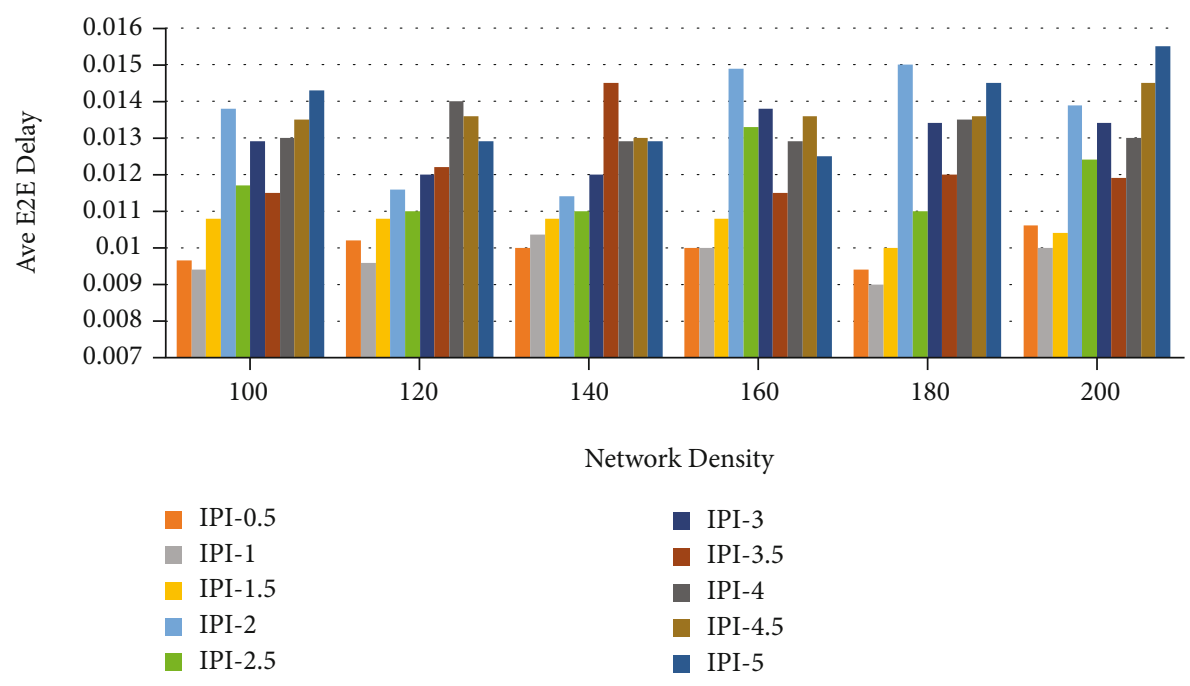

Figure 13: Average end-to-end delay over 100 to 200 nodes.

However, a slight decrease in PDR when payload increase from 64 bytes to 512 bytes suggests that other network factors other than payload negatively affect the network's performance. The graph of PDR versus node densities presented in Figure 15 shows the effect of the payload sizes on the network.

The average end-to-end delay on payload sizes seen in Figure 16 shows that larger payload sizes recorded higher delays than smaller payload sizes. 1024 bytes had the highest average delay of 19.405 , while the payload size of 32 bytes had an average delay of $13.25 \mathrm{~ms}$ for packet sizes between 100 and 200 nodes. Interestingly, 64 and 128 bytes recorded the slightest delay for all network densities, with average delays of $10.93 \mathrm{~ms}$ and $10.88 \mathrm{~ms}$. The increase in delay results from the rise in overall packets in the network per second. An increase in packets increases collisions, causing a doubling of the contention window after retransmissions.
Larger contention window sizes increase the overall network end-to-end delay.

The graph trends recorded in Figure 17 confirm the energy consumption values recorded in [22, 23]. The authors asserted that the increase in payload size increases the energy consumption of the sensor network. Payload size of 1024 bytes experiences the highest energy consumption. On the other hand, the payload size of 32-byte recorded the highest remaining energy and the least remaining energy on the individual nodes. The highest remaining energy is recorded at 140 nodes and sees a slight decline when it reaches the lowest at the same $5.58 \mathrm{~J}$ at the payload of 1024-byte.

When the number of packets generated per second increases in the network, the number of collisions increases. After a saturation point of about 140 nodes transmitting per second, the contention window of the CSMA/CA reaches its 


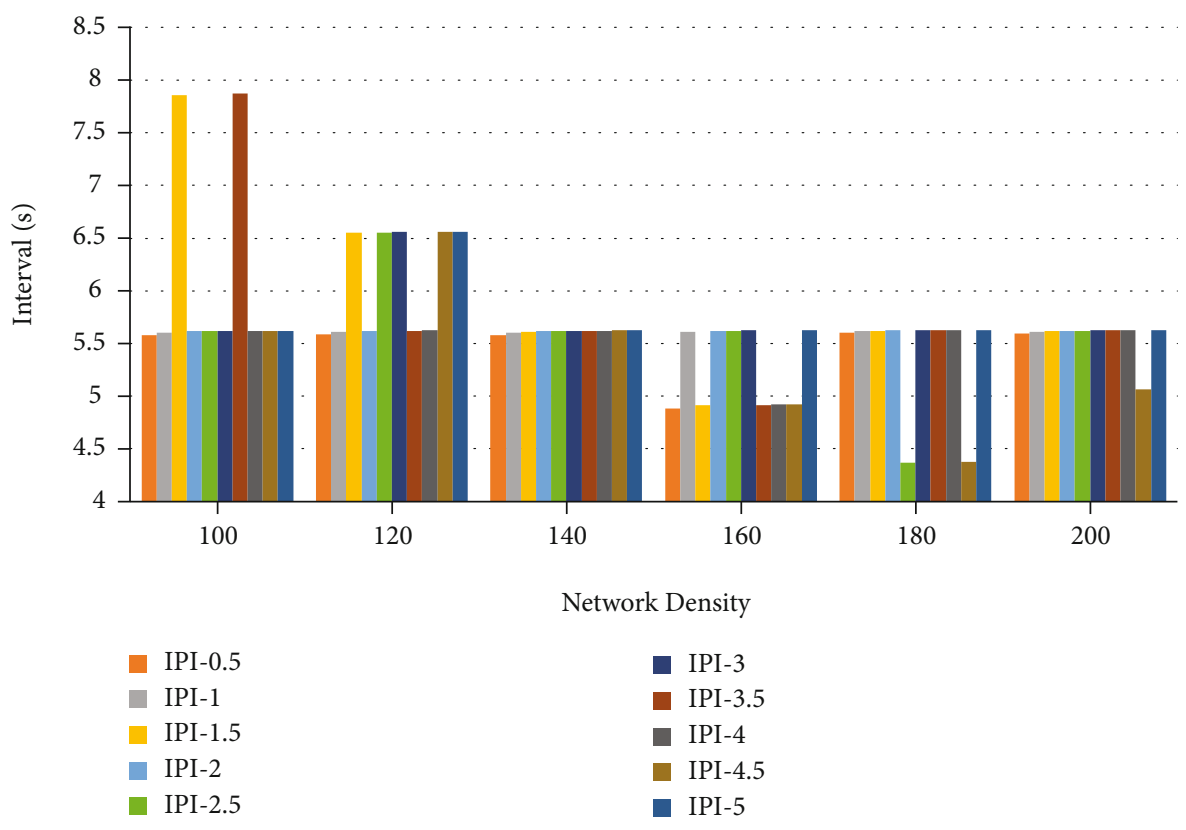

FIGURE 14: Average remaining energy on individual nodes in the network for different interpacket generation intervals.

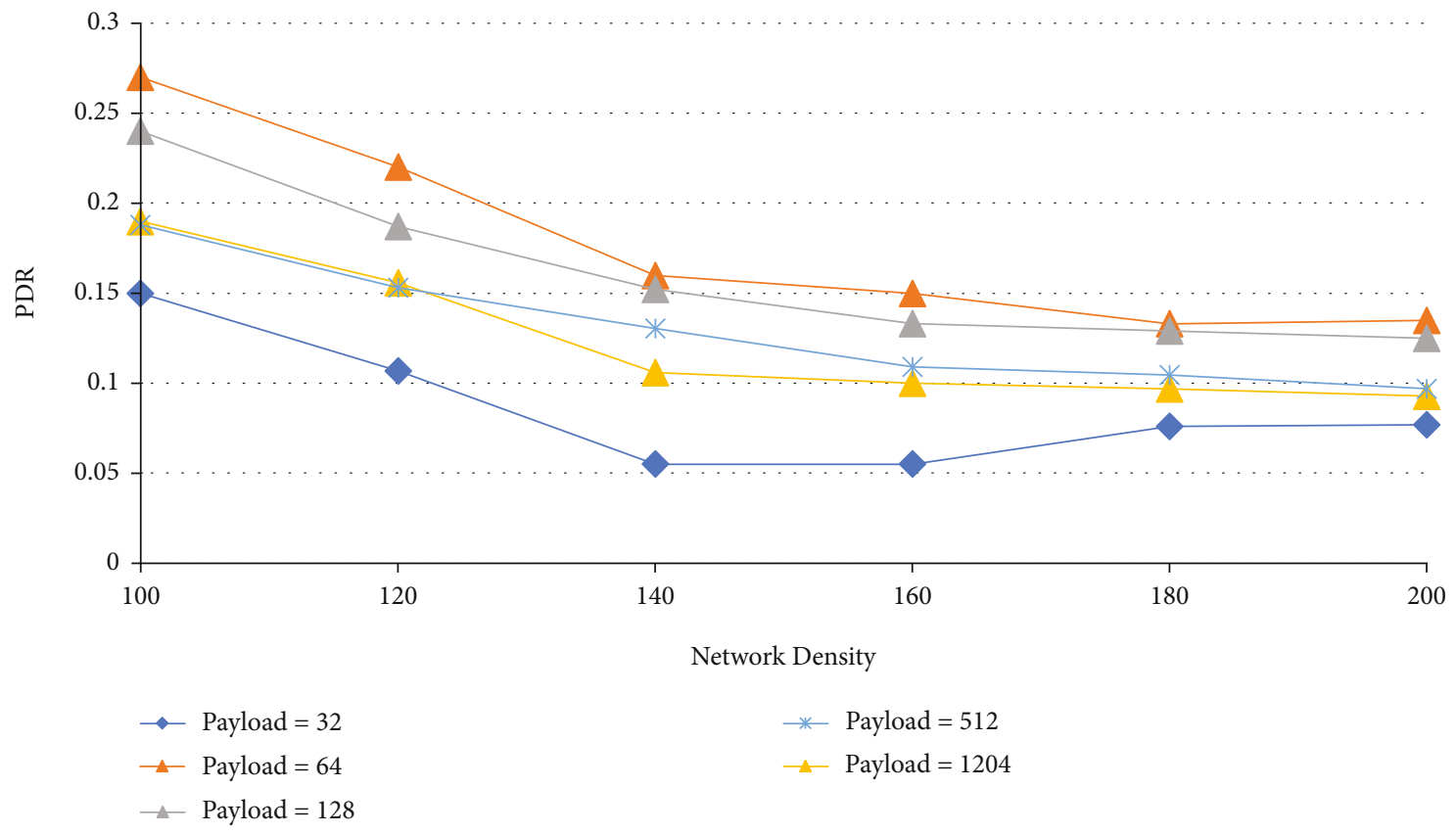

FIGURE 15: The effect of packet sizes on the packet delivery ratio.

maximum and, consequently, the maximum random backoff. After several retransmissions, the energy on a node in the network has less than their minimum threshold of energy. Further increase in packets per unit time does not increase the contention window; hence, delay remains relatively constant. Other factors like packet transmission mainly contribute to the further decrease in remaining energy. Observation of the graph for average end-to-end delay shows that after 180 nodes, the remaining energy increases slightly for all payloads and remains relatively constant for increasing network density.

As presented in Table 3, IoT and WSN applications that require continuous monitoring of the environment but are energy constrained may use these sensor node and channel parameters for packet scheduling. Applications such as animal tracking, water quality monitoring, and vehicular monitoring among others [24], precision agriculture and underground applications [25] for continuous monitoring 


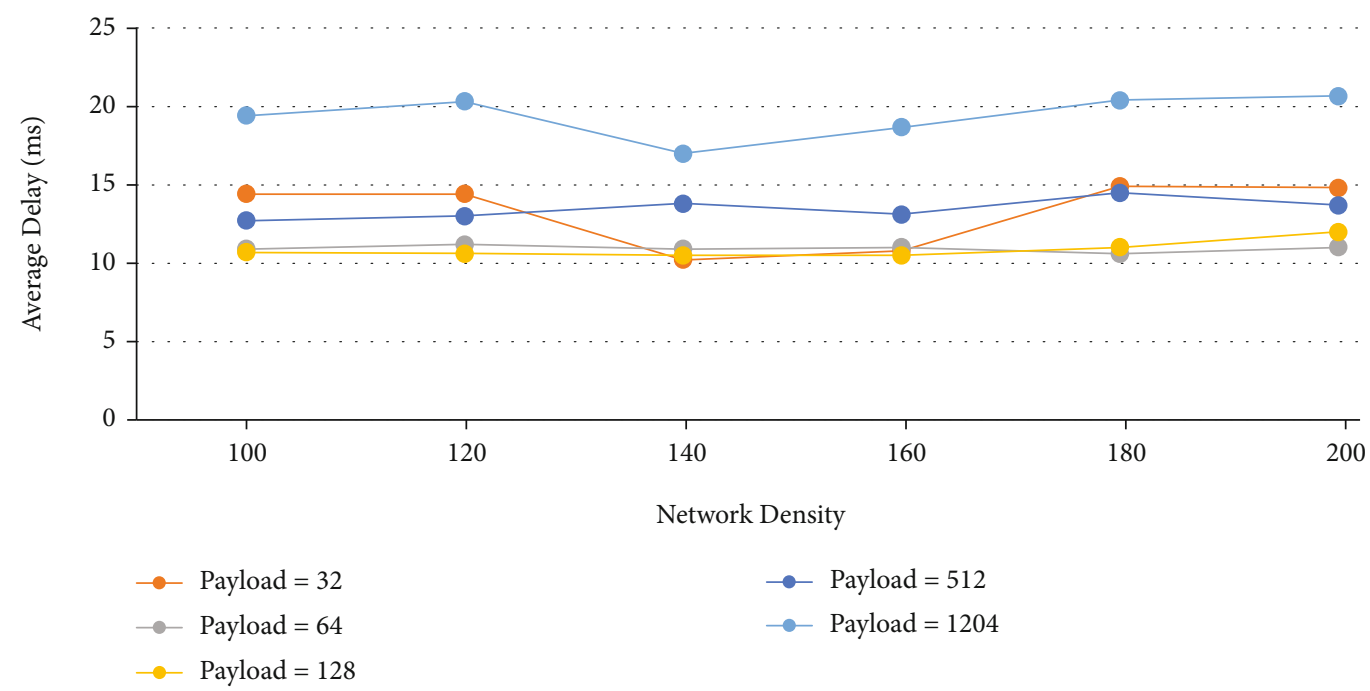

Figure 16: Average end-to-end delay of different payload sizes in IEEE802.11b.

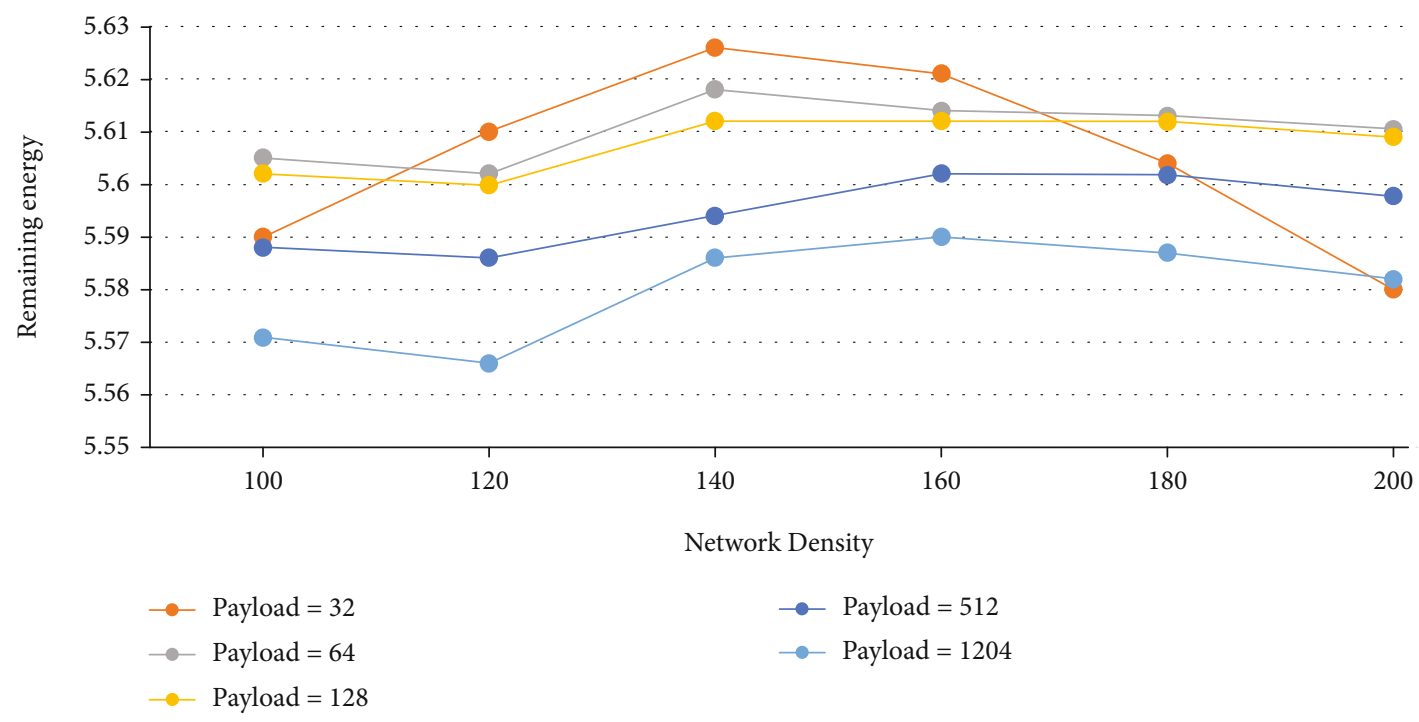

FIGURE 17: Average remaining energy for IEEE802.11b for different payload sizes.

TABLE 3: Optimal values for packet scheduling in IEEE802.11b.

\begin{tabular}{lc}
\hline Parameter & Optimal value \\
\hline Packet size of data & 64 bytes \\
Data rate & $11 \mathrm{Mbps}$ \\
Interpacket generation interval & 4 seconds \\
\hline
\end{tabular}

have data streams with spatiotemporal properties that suggest that time series models for predicting future values are beneficial for energy savings and optimum network conditions.

The importance of transmission scheduling is seen in real-time applications such as water quality, structural, air quality, animal habitat monitoring, and tracking of endangered species. These applications, for continuous monitoring, have implementation challenges such as cost, energy efficiency, and communication issues. Water quality moni- toring, with a real-time deployment discussed in the paper by [4], will receive continuous supply of the freshwater data without continuous transmission from the nodes. This work is similar to the implementation in Fiji [26], where nodes transmit continuously for an hour and sleep for 15 minutes. However, using the spatiotemporal difference between consecutive data streams, nodes here will transmit within a lesser period and, without turning nodes to sleep, achieve higher energy and network performance gains.

\section{Conclusion}

Scheduling of packets generated from sensor nodes influences transmission. In this paper, we performed sensitivity analysis of the IEEE $802.11 \mathrm{~b}$ to determine the suitable parameters to adopt when used for IoT systems. From our simulation, we observed that nodes transmit packet immediately after packet generation without significant delay. The 
effect of the scheduled packet transmission on the network was tested to determine the optimal threshold values in relation to (1) varying the inter-packet transmission interval, (2) varying the packet size, (3) and the data rate. Our findings revealed that for IoT systems implemented on IEEE802.11b, attention should be paid to the size of the payload and the data rate. These parameters have detrimental effect on the energy consumed by IoT devices and the node density. We can conveniently conclude that the optimal values obtained in Table 3 are suitable for IEEE802.11b channels where continuous monitoring with reduced data streams is essential for energy optimization.

\section{Data Availability}

The authors confirm that the data supporting the findings of this study are available within the article and its supplementary materials.

\section{Conflicts of Interest}

The authors declare that there is no conflict of interest regarding the publication of this paper.

\section{References}

[1] W. Li, F. C. Delicato, and A. Y. Zomaya, "Adaptive energyefficient scheduling for hierarchical wireless sensor networks," ACM Transactions on Sensor Networks, vol. 9, no. 3, pp. 1-34, 2013.

[2] S. Kumar and H. Kim, "Energy efficient scheduling in wireless sensor networks for periodic data gathering," IEEE Access, vol. 7, pp. 11410-11426, 2019.

[3] F. Engmann, K. S. Adu-Manu, J.-D. Abdulai, and F. A. Katsriku, "Applications of prediction approaches in wireless sensor networks," in Wireless Sensor Networks-Design, Deployment and Applications, IntechOpen, 2021.

[4] K. S. Adu-Manu, F. A. Katsriku, J.-D. Abdulai, and F. Engmann, "Smart river monitoring using wireless sensor networks," Wireless Communications and Mobile Computing, vol. 2020, Article ID 8897126, 19 pages, 2020.

[5] F. Alfayez, M. Hammoudeh, and A. Abuarqoub, "A Survey on MAC Protocols for Duty-cycled Wireless Sensor Networks," Procedia Computer Science, vol. 73, pp. 482-489, 2015.

[6] S. Kumar and S. Chauhan, "A survey on scheduling algorithms for wireless sensor networks," International Journal of Computers and Applications, vol. 20, no. 5, pp. 7-13, 2011.

[7] R. C. Carrano, D. Passos, L. C. S. Magalhaes, and C. V. N. Albuquerque, "Survey and taxonomy of duty cycling mechanisms in wireless sensor networks," IEEE Communication Surveys and Tutorials, vol. 16, no. 1, pp. 181-194, 2014.

[8] F. Engmann, F. A. Katsriku, J.-D. Abdulai, K. S. Adu-Manu, and F. K. Banaseka, "Prolonging the lifetime of wireless sensor networks: a review of current techniques," Wireless Communications and Mobile Computing, vol. 2018, Article ID 8035065, 23 pages, 2018.

[9] C. Liu, K. Wu, and J. Pei, "An energy-efficient data collection framework for wireless sensor networks by exploiting spatiotemporal correlation," IEEE Transactions on Parallel and Distributed Systems, vol. 18, no. 7, pp. 1010-1023, 2007.
[10] A. R. Kumar, "Smart network access for 802.11 based internet of things," in 2015 International Conference on Control, Instrumentation, Communication and Computational Technologies (ICCICCT), pp. 315-320, Kumaracoil, India, 2015.

[11] R. Gomathi and N. Mahendran, "An efficient data packet scheduling schemes in wireless sensor networks," in 2nd International Conference on Electronics and Communication Systems, ICECS 2015, pp. 542-547, Coimbatore, India, 2015.

[12] J. A. Khan, H. K. Qureshi, and A. Iqbal, "Energy management in wireless sensor networks: a survey," Computers and Electrical Engineering, vol. 41, pp. 159-176, 2015.

[13] O. Khader, A. Willig, and A. Wolisz, "Distributed wakeup scheduling scheme for supporting periodic traffic in WSNs," in 2009 European Wireless Conference, Aalborg, Denmark, 2009.

[14] W. Ye, J. Heidemann, and D. Estrin, "Medium access control with coordinated adaptive sleeping for wireless sensor networks," IEEE/ACM Transactions on Networking, vol. 12, no. 3, pp. 493-506, 2004.

[15] B. N. Bhandari, R. V. R. Kumar, R. Banjari, and S. L. Maskara, "Sensitivity of the IEEE $802.11 \mathrm{~b}$ MAC protocol performance to the various protocol parameters," in 2004 International Conference on Communications, Circuits and Systems (IEEE Cat. No. 04EX914), Chengdu, China, 2004.

[16] D. Saha, M. R. Yousuf, and M. A. Matin, "Energy efficient scheduling algorithm for S-MAC protocol in wireless sensor network," International Journal of Wireless \& Mobile Networks, vol. 3, no. 6, pp. 129-140, 2011.

[17] F. Royo, M. Lopez-Guerrero, T. Olivares, and L. Orozco-Barbosa, "A fast network configuration algorithm for TDMA wireless sensor networks," EURASIP Journal on Wireless Communications and Networking, vol. 2010, Article ID 513527, 10 pages, 2010.

[18] A. P. Jardosh, K. N. Ramachandran, K. C. Almeroth, and E. M. Belding-Royer, "Understanding congestion in IEEE 802.11 b wireless networks," in Proceedings of the 5th ACM SIGCOMM conference on Internet Measurement, Berkeley, CA, 2005.

[19] S. Kurt, H. U. Yildiz, M. Yigit, B. Tavli, and V. C. Gungor, "Packet size optimization in wireless sensor networks for smart grid applications," IEEE Transactions on Industrial Electronics, vol. 64, no. 3, pp. 2392-2401, 2016.

[20] M. Yigit, H. U. Yildiz, S. Kurt, B. Tavli, and V. C. Gungor, “A survey on packet size optimization for terrestrial, underwater, underground, and body area sensor networks," International Journal of Communication Systems, vol. 31, no. 11, article e3572, 2018.

[21] M. C. Vuran and I. F. Akyildiz, "Cross-layer packet size optimization for wireless terrestrial, underwater, and underground sensor networks," in IEEE INFOCOM 2008 - The 27th Conference on Computer Communications, Phoenix, AZ, USA, 2008.

[22] H.-Y. Zhou, D.-Y. Luo, Y. Gao, and D.-C. Zuo, "Modeling of node energy consumption for wireless sensor networks," Wireless Sensor Network, vol. 3, no. 1, pp. 18-23, 2011.

[23] I. Koutsopoulos and M. Halkidi, "Distributed energy-efficient estimation in spatially correlated wireless sensor networks," Computer Communications, vol. 45, pp. 47-58, 2014.

[24] K. S. Adu-Manu, C. Tapparello, W. Heinzelman, F. A. Katsriku, and J. D. Abdulai, "Water quality monitoring using wireless sensor networks," ACM Transactions on Sensor Networks, vol. 13, no. 1, pp. 1-41, 2017. 
[25] K. S. Adu-Manu, N. Adam, C. Tapparello, H. Ayatollahi, and W. Heinzelman, "Energy-harvesting wireless sensor networks (EH-WSNs)," ACM Transactions on Sensor Networks, vol. 14, no. 2, pp. 1-50, 2018.

[26] A. N. Prasad, K. A. Mamun, F. R. Islam, and H. Haqva, "Smart water quality monitoring system," in 2015 2nd Asia-Pacific World Congress on Computer Science and Engineering (APWC on CSE), Nadi, Fiji, 2016. 